\title{
Femtogram per Milliliter per Microgram per Day
}

National Cancer Institute

\section{Source}

National Cancer Institute. Femtogram per Milliliter per Microgram per Day. NCI

Thesaurus. Code C119342.

A unit of concentration equal to femtogram per milliliter, divided by micrograms per day. 